\title{
In situ Crosslinking of Nanostructured Block Copolymer Microparticles in Supercritical Carbon Dioxide
}

\author{
Guping He, ${ }^{1}$ Thomas M. Bennett, ${ }^{1}$ Kartini Alias, ${ }^{1}$ Long Jiang, ${ }^{2}$ Simon T. \\ Schwab, ${ }^{1}$ Mohammad Alauhdin, ${ }^{1}$ Steven M. Howdle ${ }^{1, *}$ \\ ${ }^{1}$ School of Chemistry, University of Nottingham, Nottingham, NG7 2RD, United Kingdom \\ ${ }^{2}$ Interface and Surface Analysis Centre, University of Nottingham, Nottingham, NG7 2RD, \\ United Kingdom
}

\begin{abstract}
We report a novel and facile approach to "fix" the internal nanostructure of block copolymer (BCP) microparticles via in situ crosslinking copolymerisation in dispersion in supercritical $\mathrm{CO}_{2}\left(\mathrm{scCO}_{2}\right)$. By delaying the addition of the crosslinker and a portion of the second monomer, polymerisation induced microphase separation (PIMS) within the microparticles is well preserved, while the growing chains of precursor poly(methyl methacrylate)-block-poly(4-vinyl pyridine) (PMMA-b-P4VP) or poly(methyl methacrylate)-block-poly(benzyl methacrylate) (PMMA- $b$-PBzMA) microparticles are crosslinked. The unique structure of the as-synthesised crosslinked microparticles were fully characterised using transmission electron microscopy (TEM), scanning electron microscopy (SEM) and atomic force microscopy (AFM). Moreover, the swelling and solubility behaviour of the crosslinked PMMA- $b$-P4VP microparticles was investigated. Notably, the porosity generated by swelling in ethanol can be well controlled by the quantity of crosslinker incorporated. Macropores $>100 \mathrm{~nm}-\sim 20 \mathrm{~nm}$, sub-10 $\mathrm{nm}$ mesopores, and non-porous microparticles were all achieved by varying the crosslinker incorporation from $0,0.5,1$, to $4 \mathrm{wt} \%$, respectively. In situ AFM nanomapping of the crosslinked P4VP domains in $80 \%$ humidity revealed that microparticles with a high degree of crosslinking (8 wt \% divinylbenzene) are highly resistant to swelling in humidity, by contrast to their non-crosslinked counterparts. This versatile approach further expands the available repertoire for fabricating porous $\mathrm{BCP}$
\end{abstract}


microparticles with tunable physico-chemical properties, morphologies and pore sizes, greatly broadening their application potential to more diverse fields.

Keywords: in-situ crosslinking, block copolymer microparticles, RAFT dispersion polymerisation, polymerisation induced phase separation, porosity control

\section{Introduction}

Block copolymer (BCP) microparticles with internal nanostructures, typically formed via microphase separation, have received considerable interest as functional materials over the last decade. Their broadening scope of application now includes drug delivery, ${ }^{1-3}$ diagnostics,${ }^{4}$ hydrogel actuators,${ }^{5}$ and impact modifiers. ${ }^{6}$ Further processing of nanostructured BCP particles can also offer hierarchically porous materials for protein separation, ${ }^{7}$ chromatographic columns and catalyst supports. Nanostructured BCP microparticles can also be loaded with inorganic nanoparticles or used as templates to direct structure formation in metal oxides, widening their applicability into other multidisciplinary areas such as energy storage, ${ }^{8,9}$ plasmonics, ${ }^{10}$ and photonics. ${ }^{11}$

Despite recent advances in the synthesis of nanostructured BCP microparticles, in particular heterogeneous controlled radical polymerisation, ${ }^{12-17}$ there is a significant intrinsic dilemma: the structural integrity of the particles can be lost in some application circumstances. For example, upon exposure to the highly dynamic and complex environments of biological fluids, ${ }^{18,19}$ or in the presence of surfactants or organic solvents or high shear forces. ${ }^{20,21}$ The stabilisation of these structures, i.e. locking them in place via mechanisms such as chemical cross-linking, could overcome these issues by improving the structural robustness of the polymer particles. ${ }^{22-24}$

To date, several experimental methods have been developed for crosslinking polymer particles. These can be divided into two approaches: post-polymerisation chemical reactions or in situ cross-linking via copolymerisation with a comonomer that has two (or more) reactive sites. The former approach involves the pre-synthesis of polymers or polymer particles with reactive groups, followed by chemical reactions with a crosslinker. $\mathrm{Xu}$ et. al showed that pre-polymerised tri-block copolymer unimers with amine groups reacted with a dialdehyde crosslinker and formed shell-crosslinked micelles. ${ }^{25}$ Furthermore, Qui and co-workers were the first to demonstrate that nano-objects 
carrying aldehyde groups formed via PISA can be crosslinked with butanediamine, producing core-crosslinked nano-objects with preserved morphologies. ${ }^{26}$ Postpolymerisation crosslinking can also be achieved via transition metal complexation ${ }^{27}$ or a sol-gel reaction. ${ }^{28}$ One of the advantages of post-polymerisation cross-linking is that it can potentially be applied to a pre-established synthesis route to precursor $\mathrm{BCP}$ particles, for which morphology and/or particle size control has already been achieved. ${ }^{29}$ It also enables polymer particles with varied chemical or physical properties to be pre-produced and stored for subsequent crosslinking reactions at a later date. However, there are drawbacks. Postpolymerisation crosslinking involves multiple steps: the synthesis and purification of precursor particles, re-dispersion or dissolution, and purification again after crosslinking, all of which results in more time and higher costs, and would not translate well to industrial production.

A second approach, in situ crosslinking, uses divinyl comonomers to form covalent crosslinks during the polymerisation, and presents an attractive alternative. The structural stabilisation of PISA-generated nano objects has been recently investigated via in situ cross-linking by copolymerisation with a divinyl comonomer. Unfortunately, the chain mobility of a growing polymer is significantly reduced upon crosslinking, which usually disrupts the copolymerisation through macro-gelation and/or impedes morphology evolution. ${ }^{30}$ To solve this problem, the Armes group explored an approach of delayed crosslinker addition for the in situ cross-linking of vesicles with a symmetric divinyl comonomer. ${ }^{21,31}$ Ethylene glycol dimethacrylate (EGDMA) was added after the consumption of the core-forming monomer to achieve a highly cross-linked third block. The delayed addition of a crosslinker pushes the crosslinking forward to a much higher degree while preserving the control over the polymerisation and particle morphology. An alternative approach uses an asymmetric cross-linker, generally bearing two vinyl groups of different reactivity, that is added prior to the commencement of the polymerisation. ${ }^{32} \mathrm{Qu}$ and co-workers developed an in situ crosslinking strategy to stabilise higher order morphologies, such as vesicles, using the synthesised asymmetric cross-linker allyl acrylamide (ALAM) ${ }^{32}$ In this way, vesicles with a higher degree of crosslinking ( $2-5$ mol\% ALAM) were achieved compared to those using the symmetric crosslinker N, N'-methylene bisacrylamide (BIS) (1 mol\%). They later proved that these crosslinked vesicles preserve RAFT end group livingness for further chain extension into triblock copolymer vesicles. ${ }^{33}$ 
Previously, we reported RAFT controlled dispersion polymerisation in $\mathrm{scCO}_{2}$ as a unique and facile route to create multigram quantities of nanostructured microparticulate BCPs. ${ }^{14,34,35}$ Moreover, we have also demonstrated that the sizes of the microparticles can be controlled reproducibly from $300 \mathrm{~nm}$ through to $5 \mu \mathrm{m} .{ }^{36}$ Very recently, we developed a facile and versatile approach to convert these nanostructured microparticles into porous microparticles via swelling/deswelling. ${ }^{34}$ It was demonstrated that the porosity can be tailored over a wide size range from 20 to 200 $\mathrm{nm}$, and diverse morphologies from isolated spherical pores, short porous channels, to interpenetrated pore networks, could be achieved by adjusting the block ratio and block length. However, in order to further exploit these materials for applications such as drug delivery, chromatographic columns, and as templates for inorganic materials, etc., it is important to consider that the particle structures and/or scaffolds can be susceptible to collapse in the presence of organic solvents. Little attention has been focused on the internal crosslinking of such larger micron-sized particles, particularly in the context of those with hierarchical structures achieved in situ through polymerisation induced microphase separation.

On the basis of these considerations, we report a method to selectively crosslink the internal phase separated domains of BCP microparticles during their one-pot polymerisation in $\mathrm{scCO}_{2}$, providing a means to bolster their structural integrity in the presence of solvents, or indeed, any other stimuli (Scheme 1). To the best of our knowledge, this is the first time that nanostructured BCP microparticles have been crosslinked during polymerisation in-situ without compromising their polymerisation induced phase separated morphologies.

\section{Experimental Section}

\subsection{Materials}

Methyl methacrylate (MMA, Fisher, >99\%), 4-vinylpyridine (4VP, Acros, 99\%) and benzyl methacrylate (BzMA, Sigma-Aldrich, 98\%) were purified by passing through a neutral alumina column and stored at $-20{ }^{\circ} \mathrm{C}$. 2-(dodecylthiocarbonothioylthio)-2methylpropionic acid (DDMAT) was synthesised following a literature procedure. ${ }^{37} \alpha$ azobis(isobutyronitrile) (AIBN, Wako, 97\%) was purified by recrystallisation in methanol. The dispersion stabiliser poly(dimethylsiloxane)-monomethyl methacrylate 
(PDMS-MA, ABCR, $M_{\mathrm{n}}=10,000 \mathrm{~g} / \mathrm{mol}$ ), divinylbenzene (DVB, Sigma-Aldrich, $85 \%$ ), ethylene glycol dimethacrylate (EGDMA, Sigma-Aldrich, 98\%), $\mathrm{CDCl}_{3}$ (Aldrich, 99.9\%), HPLC grade THF (Acros), chloroform (Aldrich, 99.9\%), triethylamine (Acros, 99.9\%) and iodine (Fisher) were used as received. Agar 100 resin (Agar Scientific) was used as received, and a formulation of medium hardness was used for embedding samples.

\subsection{Two-stage in situ crosslinking of PMMA-b-P4VP microparticles via RAFT dispersion copolymerisation in $\mathrm{scCO}_{2}$}

The synthesis of in situ crosslinked PMMA- $b$-P4VP microparticles is a modified procedure based on our earlier methods ${ }^{14,} 38$ for synthesising non-crosslinked BCP microparticles. A typical one-pot and two-steps synthesis is described below for PMMA50- $\boldsymbol{b}$-P4VP17- $\boldsymbol{b}$-P4VP16/D1 (1 wt\% of crosslinker relative to the total 4VP monomer), where the subscripts denote the target molecular weight values in $\mathrm{kg} / \mathrm{mol}$. A high-pressure stainless steel autoclave $(60 \mathrm{ml})$ was flushed with $\mathrm{CO}_{2}(5 \mathrm{MPa})$ for 15 min before adding the reactants to synthesise the first block. MMA (7.5 g), DDMAT RAFT agent (55 mg), AIBN initiator $(25 \mathrm{mg})$, and PDMS-MA surfactant (0.625 g) were pre-mixed in a glass vial and purged with argon for $20 \mathrm{~min}$ before transfer into the autoclave with a glass syringe. The $\mathrm{CO}_{2}$ pressure in the autoclave was first increased to $\sim 6 \mathrm{MPa}$ and the autoclave was then heated to $60{ }^{\circ} \mathrm{C}$ while the contents were stirred mechanically. ${ }^{39}$ The final pressure and temperature were gradually adjusted to $\sim 27 \mathrm{MPa}$ and $65{ }^{\circ} \mathrm{C}$ over a period of $15 \mathrm{~min}$. The reaction was carried out for $18 \mathrm{~h}$ to achieve full conversion of the MMA. The first portion of 4VP (2.55 g) and additional AIBN (6.3 $\mathrm{mg}$ ) were purged with Argon for $15 \mathrm{~min}$ and added to the autoclave via a HPLC pump at $1 \mathrm{ml} / \mathrm{min}$. The polymerisation of $4 \mathrm{VP}$ was allowed to proceed for $20 \mathrm{~h}$, followed by addition of the second portion of 4VP (2.45 g) and AIBN (6.3 mg) together with DVB $(0.05 \mathrm{~g})$ in the same way. The crosslinking copolymerisation of 4VP with DVB was allowed to proceed for $20 \mathrm{~h}$. The autoclave was first cooled to $25{ }^{\circ} \mathrm{C}$ and then depressurised. The product was observed to be a fine off-white powder $(\sim 9 \mathrm{~g})$ and was collected for analysis. 

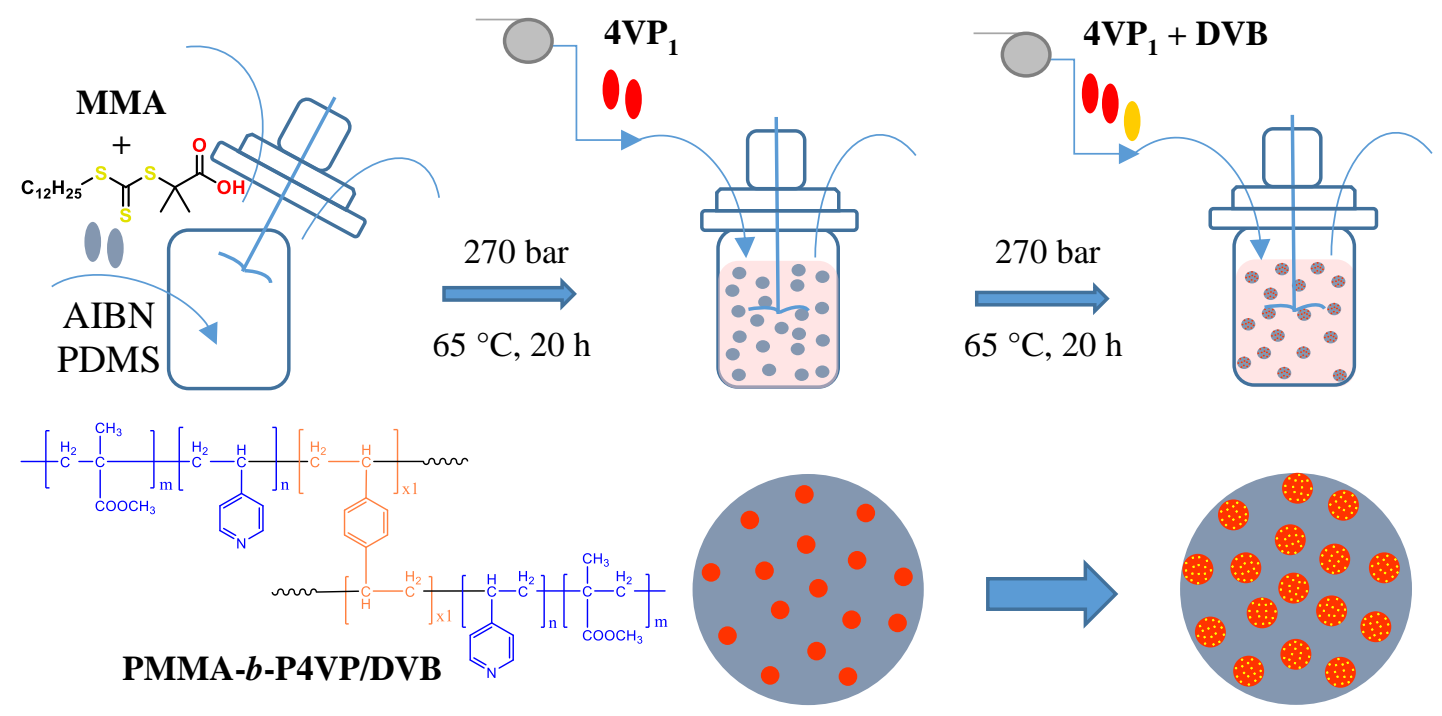

Scheme 1. The in situ crosslinking of PMMA- $b$-P4VP microparticles by RAFT dispersion polymerisation in $\mathrm{scCO}_{2}$ in a one-pot, two-step process.

\subsection{Two-stage in situ crosslinking of PMMA-b-PBzMA microparticles via RAFT dispersion copolymerisation in $\mathrm{scCO}_{2}$}

The synthesis of in situ crosslinked PMMA- $b$-BzMA microparticles proceeded as described for the PMMA- $b$-P4VP BCPs up until the addition of the second monomer. The first portion of BzMA (7.5 g) and additional AIBN $(6.3 \mathrm{mg})$ was then purged with Argon for $15 \mathrm{~min}$ and added to the autoclave via a HPLC pump at $1 \mathrm{ml} / \mathrm{min}$. The polymerisation of BzMA was allowed to proceed for 48 hours, followed by addition of the second portion of BzMA (2.5 g) and AIBN (6.3 mg), together with EGDMA (0.05 $\mathrm{g}$ ) in the same way. The crosslinking copolymerisation of PBzMA with EGDMA was allowed to proceed for $24 \mathrm{~h}$. The autoclave then cooled to $25{ }^{\circ} \mathrm{C}$ and subsequently depressurised. The product was observed to be a fine off-white powder $(16.4 \mathrm{~g})$ and was collected for analysis.

\subsection{Porosity control by degree of crosslinking}

The swelling process of the PMMA- $b$-P4VP BCP microparticles in ethanol followed our earlier published procedures. ${ }^{34}$ The original BCP microparticles $(50 \mathrm{mg})$ were fully dispersed in alcohol $(3 \mathrm{ml})$ in a glass vial $(3.5 \mathrm{ml})$ and shaken 3 times by hand over a two-hour period. The particles were then left standing until they settled to the bottom of the vial. The upper alcohol layer was removed and then hexane $(3 \mathrm{ml})$ was poured into the vial and the particles were fully rinsed by vigorous shaking. The particles were 
then allowed to resettle before removing the hexane layer after $2 \mathrm{~h}$. The hexane rinse was repeated three more times to completely remove all of the alcohol and the polymer was dried in a vacuum oven at $25^{\circ} \mathrm{C}$ for $>2 \mathrm{~h}$ before further analysis.

\subsection{Analysis}

The non-crosslinked BCPs were characterised by ${ }^{1} \mathrm{H}$ NMR in $\mathrm{CDCl}_{3}$ on a Bruker AV3400 (400 MHz) spectrometer. Gel Permeation Chromatography (GPC) analysis was carried out on an Optilab-rEX (Wyatt) in a mixture of chloroform/ethanol/triethylamine (90/10/1 by volume) for non-crosslinked PMMA- $b$ $\mathrm{P} 4 \mathrm{VP}$ at a flow rate of $0.5 \mathrm{~mL} / \mathrm{min}$ and $25{ }^{\circ} \mathrm{C}$. GPC Columns were composed of a $\mathrm{K}$ G, a K-805L and a HT-803 column (Shodex) and calibrated with narrow PMMA standards.

For SEM, the samples were mounted on an aluminium stub with a carbon tab and sputter-coated with platinum or iridium prior to imaging on a JEOL 7100F FEG-SEM at accelerating voltage of $2 \mathrm{kV}$, or a FEI Quanta 650 ESEM at $10 \mathrm{kV}$. The average pore widths and microparticle diameters based on SEM was measured by counting over 100 pores or microparticles using the commercial software package NanoMeasurer 1.2.5.

Thin sections for cross-sectional TEM imaging were obtained from the original powder BCP microparticulate samples by embedding them in an epoxy resin (Agar 100) cured at $55{ }^{\circ} \mathrm{C}$ for 2 days. Thin sections $(\sim 80 \mathrm{~nm})$ were cut by ultra-microtome using a diamond knife (Leica Diatome Ultra $45^{\circ}$ ) and were placed on copper TEM grids (Agar). These cross-sections on the copper grids were then imaged using a FEI Tecnai BioTwin-12 TEM at $100 \mathrm{kV}$ at room temperature. The samples were not stained, except where specified. The internal domain sizes were measured by counting over 100 domains in the TEM images using the software package NanoMeasurer 1.2.5.

AFM in situ nano-mapping of the swelling behaviour of BCP microparticle thin sections under controlled humidity was performed on a Veeco Enviroscope (eScope) AFM equipped with humidity control. Non-conductive silicon nitride cantilevers (Veeco, resonant frequency $=22 \mathrm{kHz}$, spring constant $=0.07 \mathrm{Nm}^{-1}$, tip radius $20 \mathrm{~nm}$ ) were used. Height and phase data were collected simultaneously in tapping mode. Scans were taken on fresh smooth surfaces of microparticle thin sections cut by ultramicrotomy. Imaging was performed at a $0.5-1 \mathrm{~Hz}$ scan rate. Data analysis was 
performed with the NanoScope Analysis software version x 86 .

\section{Results and Discussion}

\subsection{In situ crosslinking copolymerisation of PMMA-b-P4VP microparticles}

One-step addition. The in situ crosslinking of PMMA- $b$-P4VP was first carried out in a one-pot and one-step procedure, in which the crosslinker DVB was copolymerised along with 4VP once the first monomer, MMA, was consumed. The target block ratio of PMMA/P4VP was kept at a constant molar value of 60/40, while varying the crosslinking degree or MW (two MW series were synthesised, 50k- $b-33 \mathrm{k}$ and 200k- $b$ 133k). ${ }^{1} \mathrm{H}$ NMR spectroscopy analysis of the two non-crosslinked BCP samples confirmed that similar PMMA/P4VP molar block ratios of 56.5/43.5 and 63.1/36.9 were achieved for M50-V33 and M200-V133, respectively (SI Fig. 1a). Furthermore, GPC analysis (relative to PMMA standards) returned $M_{\mathrm{n}}$ values of $70.2 \mathrm{kDa}(\nexists=1.68)$ and $153.1 \mathrm{kDa}(\bigoplus=2.24)$ for M50-V33 and M200-V133, respectively, indicating that the RAFT agent is less effective at controlling the polymerisation when targeting higher molecular weight values (SI Fig. 1b). This result is unsurprising given that well-defined polymers with very high molecular weights are difficult to synthesise using RAFT polymerisation when using monomers with low propagation rate coefficient $\left(k_{\mathrm{p}}\right)$ values (i.e., 4VP, styrene, etc.). ${ }^{40}$ Nevertheless, the $M_{\mathrm{n}}$ value for M200-V133 is still significantly larger than that obtained for M50-V33, and although the GPC trace is broad, the molecular weight distribution is still unimodal.

When either no crosslinker or a small quantity of crosslinker was used (0.5 wt\% DVB relative to 4VP), the products (M50-V33 and M50-V33/D0.5 in Table 1) form very well defined microparticles, as illustrated by the SEM images (inset in Fig. 1a-b). TEM cross-sectional views (Fig. 1a-b) reveal that the particles have an internal spherical morphology, which confirms that the polymerisation induced phase separation was preserved. In both cases the spherical areas, recognised as the P4VP domains because they are darker than the PMMA domains in bright-field TEM,${ }^{14}$ are surrounded by a matrix of PMMA. The internal P4VP domains of M50-V33/D0.5 $\left(\mathrm{d}_{\mathrm{P} 4 \mathrm{VP}}=34 \mathrm{~nm}\right)$, are much smaller than those of the non-crosslinked M50-V33 $\left(\mathrm{d}_{\mathrm{P} 4 \mathrm{VP}}=59 \mathrm{~nm}\right)($ Table 1 and Fig. 1a,b). This size disparity is strong evidence that the P4VP domains have become crosslinked, because this will greatly restrict their capacity to be swollen by the 
remaining monomer and/or $\mathrm{scCO}_{2}$ present, on account of the additional covalent linkages between the adjacent polymer chains. ${ }^{41}$ Together, these data corroborate that the one-step crosslinking copolymerisation of 4VP with $0.5 \mathrm{wt} \%$ of DVB proceeds under RAFT control while also maintaining an adequate dispersion.

When the DVB level was increased to $1 \mathrm{wt} \%$ (M50-V33/D1 in Table 1), SEM images (SI Fig. 2a) show that the particles became severely fused and agglomerated, and many sub-100 nm particles were also formed. Nevertheless, the cross-sectional TEM images show that the microparticles themselves still preserve an internal spherical morphology (SI Fig. 2b). Notably, the small particles present as darker areas and some are fully black spheres in bright field TEM, which confirms the small particles are mainly formed from homo-P4VP chains. Thus, the crosslinking copolymerisation loses control when $1 \mathrm{wt} \%$ of DVB is added together with $4 \mathrm{VP}$ in one-step, and the dispersion becomes unstable at the latter stages of the copolymerisation.

Table 1. Crosslinked PMMA- $b$-P4VP microparticles synthesised by RAFT dispersion polymerisation in $\mathrm{scCO}_{2}{ }^{\mathrm{a}, \mathrm{b}}$

\begin{tabular}{|c|c|c|c|c|c|}
\hline Block copolymers & Target $M_{n}$ & Crosslinker & SEM & \multicolumn{2}{|c|}{ TEM } \\
\hline $\mathrm{M}-\mathrm{V} / \mathrm{D}^{\mathrm{c}}$ & $\begin{array}{l}\text { PMMA-P4VP } \\
\qquad(\mathrm{g} / \mathrm{mol})\end{array}$ & $\begin{array}{c}\mathrm{DVB} /\left(\mathrm{V}_{1}+\mathrm{V}_{2}\right. \\
)(\mathrm{wt} \%)\end{array}$ & $\begin{array}{c}d_{m} \\
(\mu \mathrm{m})\end{array}$ & $\begin{array}{c}\text { Internal } \\
\text { Morphology }\end{array}$ & $\begin{array}{l}\mathrm{d}_{\mathrm{P} 4 \mathrm{VP}}^{\mathrm{d}} \\
(\mathrm{nm})\end{array}$ \\
\hline M50-V33 & $50 \mathrm{k}-33 \mathrm{k}$ & 0 & $1.57 \pm 0.31$ & SPH & $59 \pm 8.5^{\mathrm{e}}$ \\
\hline M50-V33/D0.5 & $50 \mathrm{k}-33 \mathrm{k}$ & 0.5 & $0.74 \pm 0.17$ & SPH & $34 \pm 3.7$ \\
\hline M50-V33/D1 & $50 \mathrm{k}-33 \mathrm{k}$ & 1 & $0.40^{\mathrm{f}}$ & SPH & - \\
\hline M50-V $\mathrm{V}_{1} 17-\mathrm{V}_{2} 16 / \mathrm{D} 0.5$ & 50k-17k-16k & 0.5 & $1.48 \pm 0.25$ & SPH & $48 \pm 5.7$ \\
\hline M50-V ${ }_{1} 17-V_{2} 16 / D 1$ & 50k-17k-16k & 1 & $1.29 \pm 0.27$ & SPH & $36 \pm 4.2$ \\
\hline M50-V $V_{1} 17-V_{2} 16 / D 2$ & $50 \mathrm{k}-17 \mathrm{k}-16 \mathrm{k}$ & 2 & $1.24 \pm 0.30$ & SPH & $33 \pm 3.1$ \\
\hline M50-V ${ }_{1} 17-V_{2} 16 / D 4$ & 50k-17k-16k & 4 & $0.99 \pm 0.36$ & SPH & $29 \pm 3.3$ \\
\hline M50-V $120-V_{2} 13 / D 8$ & 50k-20k-13k & 8 & $0.89 \pm 0.29$ & SPH & $25 \pm 2.5$ \\
\hline M200-V133 & $200 \mathrm{k}-133 \mathrm{k}$ & 0 & $1.49 \pm 0.14$ & SPH & $56 \pm 8.4$ \\
\hline $\mathrm{M} 200-\mathrm{V}_{1} 80-\mathrm{V}_{2} 52 / \mathrm{D} 4$ & $200 \mathrm{~K}-80 \mathrm{~K}-52 \mathrm{~K}$ & 4 & $1.13 \pm 0.22$ & SPH & $41 \pm 7.5$ \\
\hline
\end{tabular}


${ }^{\text {a }}$ For the PMMA $1{ }^{\text {st }}$ block the molar ratio of CTA/AIBN $=1: 1$; for the chain extension of $4 \mathrm{VP}$, macro-RAFT/AIBN $=1: 0.25 \mathrm{~mol} / \mathrm{mol}$; the reaction time for PMMA is $18-24 \mathrm{~h}$ and $16-24 \mathrm{~h}$ for $\mathrm{P} 4 \mathrm{VP} / \mathrm{DVB} ;{ }^{\mathrm{b}}$ the NMR analysis of non-crosslinked $\mathrm{BCP}$ is in $\mathrm{CDCl}_{3}$; the molar block ratio of PMMA/P4VP is 56.5/43.5 for M50-V33, and is 63.1/36.9 for M200-V133; the GPC analysis of non-crosslinked BCPs was carried out in a mixture of chloroform/ethanol/triethylamine (90/10/1 by volume) with narrow PMMA standards; for M50-V33, $M_{\mathrm{n}}=70.2 \mathrm{kDa}, \nexists=1.68$; for M200-V133, $M_{\mathrm{n}}=153.1 \mathrm{kDa}, \emptyset=2.24 ;{ }^{\mathrm{c}} \mathrm{M}, \mathrm{V}$, and D denote PMMA, P4VP, and crosslinker DVB, respectively; the numbers following $\mathrm{M}$ and V denote the target MW in $\mathrm{kDa}$; the subscripts following $\mathrm{V}$ denote that $4 \mathrm{VP}$ is added sequentially in two steps; the numbers following D denote the weight percentage of DVB relative to total $4 \mathrm{VP}$; ${ }^{\mathrm{d}}$ the average domain size of P4VP ( $\left.\mathrm{d}_{\mathrm{P} 4 \mathrm{VP}}\right)$ was calculated by counting over 100 domains from TEM images; ${ }^{\mathrm{e}} \mathrm{d}_{\mathrm{P} 4 \mathrm{VP}}$ is $28 \mathrm{~nm}$ in the core area, $59 \mathrm{~nm}$ in the middle layer and $134 \mathrm{~nm}$ at periphery layer; ${ }^{\mathrm{f}}$ the microparticles are partially fused.

Two-step P4VP addition - delayed addition of crosslinker. A key challenge is to increase the proportion of crosslinker without sacrificing the microparticle structure and the internal morphology. To do this we devised an alternative two-stage addition method in which addition of the crosslinker is delayed. Specifically, the chain extension of $4 \mathrm{VP}$ first proceeds in the absence of DVB until an internal phase separated morphology has formed. This is then followed by the addition of DVB in conjunction with the remaining $4 \mathrm{VP}$ (Scheme 1). In our recent study, ${ }^{34}$ we determined that the polymerisation induced microphase separation begins when $\mathrm{P} 4 \mathrm{VP}=\sim 5 \mathrm{kDa}$ when the first PMMA-block has a length of $\sim 50 \mathrm{kDa}$ (i.e. $\sim 9$ mol\% P4VP). Herein, 4VP was added in two steps, where the first portion of 4VP was sufficient to induce microphase separation when fully polymerised (Table 1). In this way, crosslinker quantities of up to $8 \mathrm{wt} \%$ relative to the total $4 \mathrm{VP}$ monomer content (combined volume of the first and second additions) were added to the reaction, with the products being obtained as fine powders in each case. SEM shows that the product retains a homogeneous microparticle structure without inter-particle fusion or agglomeration (insets in Fig. 1c,d,f). Furthermore, a trend of decreasing microparticle diameter as a function of crosslinker incorporation was observed in size measurements from >100 microparticles (Fig. 2a). As was the case for the domain size reduction in the crosslinked samples, the decreasing diameter values stem from their reduced capacity to be swollen by the remaining monomer and/or $\mathrm{scCO}_{2}$ present. Critically, TEM analysis of the respective crosssections reveals that all of the crosslinked microparticle samples synthesised in this way 
(1, 4 and 8 wt\% DVB) retain an internal spherical morphology (Fig. 1c,d,f) that is consistent with that of their non-crosslinked analogues (Fig. 1a,e) (note that the SEM and TEM images for samples M50-V117-V $\mathbf{2}$ 16/D2 and M50-V117-V $V_{2} 16 / D 4$ can be found in SI Fig 3a,b). In each case the average domain size of the crosslinked P4VP is also smaller than for those without crosslinker, for reasons discussed previously (Fig. 1 and Table 1). This new approach allows us to achieve extremely high crosslinking levels; up to $8 \mathrm{wt} \%$ of DVB relative to the total $4 \mathrm{VP}$ without any noticeable compromise of the internal microphase separation using our delayed addition method (Fig. 1d).
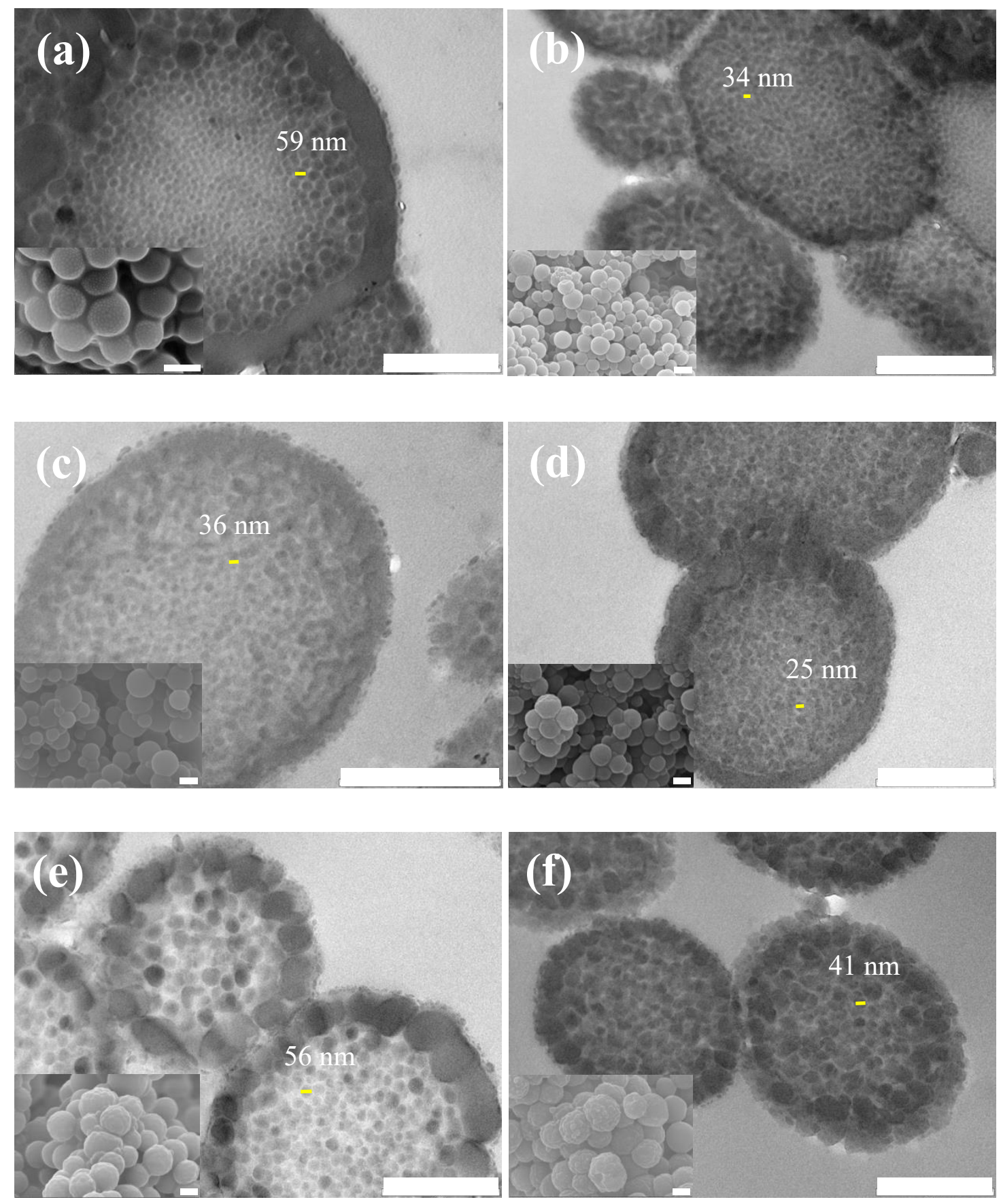
Figure 1. TEM cross-sectional views of the in situ crosslinked PMMA- $b$-P4VP microparticles with varied degrees of crosslinking. (a) non-crosslinked M50-V33; (b) crosslinked M50V33/D0.5 with 0.5 wt $\%$ crosslinker DVB; (c) crosslinked M50-V $\mathbf{1} 17-V_{2} \mathbf{1 6} / \mathrm{D} 1$ with 1 wt $\%$ DVB; (d) crosslinked M50-V $\mathbf{1 2 0}-\mathbf{V}_{2} \mathbf{1 3} / \mathbf{D 8}$ with 8 wt\% DVB; (e) non-crosslinked M200-V133; (f) crosslinked M200- $V_{1} \mathbf{8 0}-V_{2} 52 / D 4$ with $4 \mathrm{wt} \%$ DVB. The samples are unstained except in (a-b), which were stained with $\mathrm{I}_{2}$ vapour for $2 \mathrm{~h}$. The insets are the corresponding SEM images of the microparticles. The scale bar represents $500 \mathrm{~nm}$ in (a-f), and $1 \mu \mathrm{m}$ in the insets.

Physical properties of crosslinked PMMA-b-P4VP. To further confirm the crosslinking within microparticles, dissolution tests were carried out. The crosslinked microparticles were first dissolved into chloroform, a good solvent for both the PMMA and P4VP blocks. Non-crosslinked M50-V33 fully dissolved in chloroform, forming a transparent solution in $<5$ min. By contrast, the microparticles crosslinked with $0.5-$ $8 \mathrm{wt} \%$ of DVB remain as a cloudy dispersion in chloroform indefinitely, as shown in (SI Fig. 4). These data suggest that the particle scaffolds collapse in chloroform but the polymers are predominantly insoluble.

The particles were then further dispersed into THF, which is also a good solvent for PMMA but only dissolves P4VP with shorter chain lengths (MW $<5000 \mathrm{kDa}$, i.e. repeating units < 45). ${ }^{42,43}$ The non-crosslinked M50-V33 microparticles collapsed and dissolved into fused vesicular-like nano-objects (Fig. 3a), which when further diluted gradually became dispersed vesicles (SI Fig. 5). The vesicles are expected to form through the dissolution and re-assembly of the BCP chains in THF. By contrast, even at a low crosslinker incorporation $(0.5 \mathrm{wt} \%$ DVB - M50-V33/D0.5) the microparticles presented good resistance to THF dissolution as revealed by TEM analysis (Fig. 3b). The microparticulate scaffold was mostly preserved, although a few particles clearly did dissolve and form nano-spheres. These results indicate that despite the crosslinking copolymerisation of $4 \mathrm{VP}$ and DVB occurring predominantly within the pre-formed P4VP domains within the microparticles, the otherwise THF soluble PMMA matrix is also imparted with a high level of solvent resistance. 

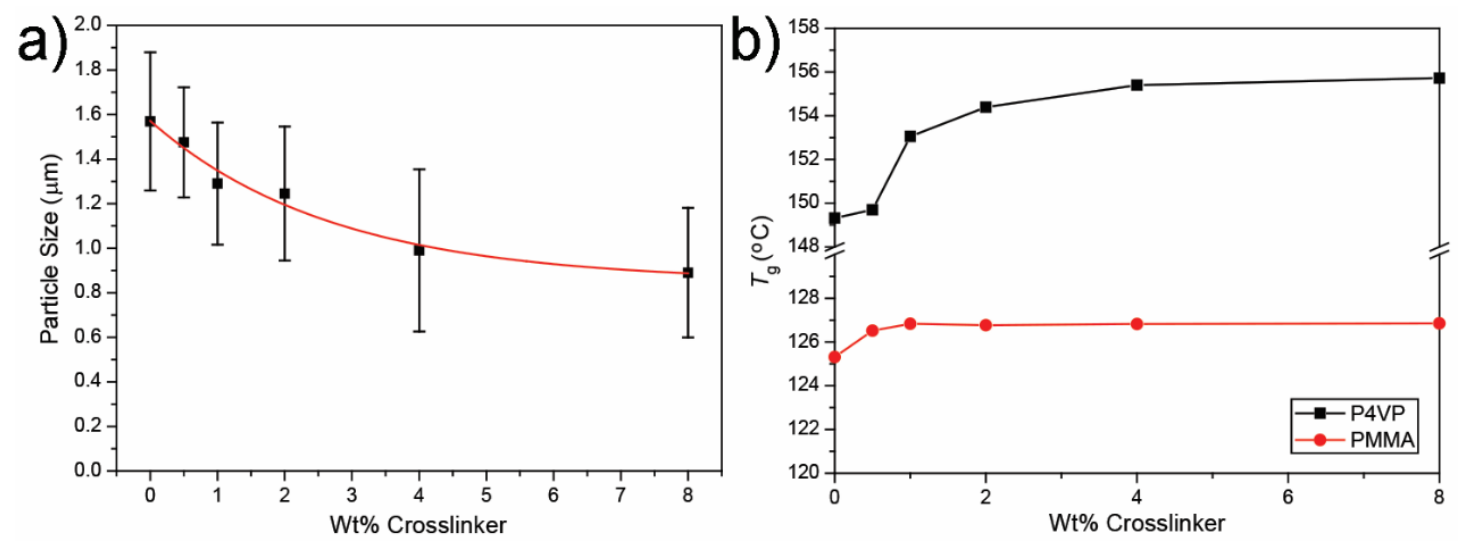

Figure 2. (a) Microparticle diameter as a function of crosslinker incorporation. The error bars are standard deviation values in the particle size measurements taken from the SEM images. The red line is an exponential fit to the data. (b) $T_{\mathrm{g}}$ values of the PMMA and P4VP blocks as a function of the DVB quantity added to the polymerisation. The data presented is for the BCPs synthesised using the two-stage crosslinking method.

The relationship between the amount of crosslinker added to the polymerisation and the $T_{\mathrm{g}}$ of each polymer block was studied using differential scanning calorimetry, for the samples synthesised using the two-stage crosslinking method (Fig. 2b, see SI Fig. 6 for the raw data). The $T_{\mathrm{g}}$ values of the PMMA domains increased slightly for the samples synthesised with $0-1 \mathrm{wt} \%$ of DVB, but remained unchanged as the quantity of DVB was increased further. The $T_{\mathrm{g}}$ of the P4VP domains also increased relatively little upon the addition of $0.5 \mathrm{wt} \% \mathrm{DVB}\left(<1{ }^{\circ} \mathrm{C}\right)$, but by contrast increased substantially over the range of $1-2 \mathrm{wt} \%$ of DVB $\left(>4{ }^{\circ} \mathrm{C}\right)$. A further increase in the P4VP $T_{\mathrm{g}}$ was observed for the sample containing $4 \mathrm{wt} \%$ of DVB, and by $8 \mathrm{wt} \%$ the value appeared to be converging upon an upper limit of $\sim 156{ }^{\circ} \mathrm{C}$. This data agrees quite well with previous studies, where it is reported that the $T_{\mathrm{g}}$ values of polymers typically increases in response to the addition of crosslinking agents. ${ }^{44,} 45$ It also indicates that the crosslinking reactions predominantly influence the mechanical properties of the P4VP domains, as expected based on the microphase separated morphology of the samples and that the crosslinker is only added after the PMMA block has been completely polymerised. 

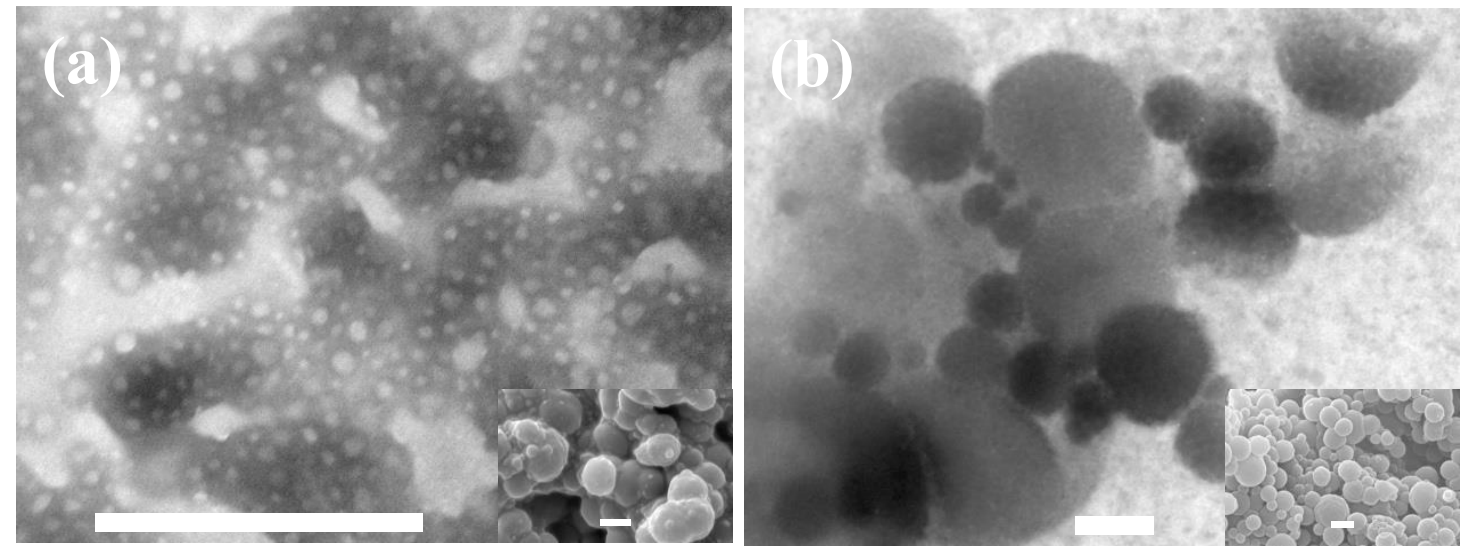

Figure 3. TEM images of non-crosslinked (a) and crosslinked microparticles (b) in THF with $50 \mathrm{mg} / \mathrm{ml}$. (a) M50-V33, (b) M50-V33/D0.5. The insets are the corresponding SEM images. The samples are unstained. The scale bars represent $1 \mu \mathrm{m}$.

\subsection{Applicability to other BCP systems and morphologies: PMMA-b-PBzMA}

To evaluate whether the two-stage synthetic protocol could also be applied to other BCP systems and/or morphologies, a second series of reactions was completed using the monomer BzMA in place of 4VP. By contrast to P4VP, the experimental phase diagram for PMMA- $b$-PBzMA in $\mathrm{scCO}_{2}$ is much closer to that predicted theoretically for a BCP in the melt state, on account of it swelling the two polymers comparably. ${ }^{46}$, ${ }^{47}$ Hence, PMMA- $b$-PBzMA BCPs with similar block lengths are expected to form a lamellar morphology, assuming their molecular weight values (and thus $\chi N$ values) are high enough to induce microphase separation.

The difunctional monomer EGDMA was instead used for the crosslinking copolymerisation of BzMA because both are of the methacrylate monomer class. Initially, a PMMA- $b$-PBzMA BCP was synthesised without the inclusion of crosslinker, targeting a length of $50 \mathrm{kDa}$ for both blocks (M50-Bz50). ${ }^{1} \mathrm{H}$ NMR spectroscopy returned a PMMA/PBzMA weight ratio value of 44.6/55.4 that was close to the target of 50/50 (SI Fig. 7a). The GPC data (relative to PMMA) also indicated that the chain extension reaction was well controlled by the RAFT agent, returning $M_{\mathrm{n}}$ values of $47.4 \mathrm{kDa}(Ð=1.42)$ and $91.8 \mathrm{kDa}(\nexists=1.45)$ for the PMMA block and final BCP, respectively (SI Fig. 7b). Critically, the SEM and TEM images revealed that a well-defined microparticle product with an internal lamellar morphology was achieved (Fig. 4a). 
A second reaction was then completed using the one-stage method, in which $0.5 \mathrm{wt} \%$ of EGDMA was added in conjunction with the BzMA (M50-Bz50/E0.5). SEM images again showed that well-defined and homogeneous microparticles had again been formed. However, TEM images of the microparticle cross-sections revealed that the BzMA domains had not formed any recognisable microphase separated morphology, instead aggregating into large unstructured internal domains with some overall resemblance to a core-shell configuration (Fig. $\mathbf{4 b}$ ). This result is further verification that the addition of crosslinker to the polymerisation greatly hinders the microphase separation process. Thus, we hypothesised that in order to achieve a crosslinked lamellar structure, the EGDMA should only be added to the reaction after both blocks had achieved equal (or very similar) values of molecular weight.

The PMMA- $b$-PBzMA synthesis was therefore repeated, targeting an adjusted final PBzMA molecular weight of $75 \mathrm{kDa}$. In this case, two-thirds of the BzMA monomer was added to the polymerisation in the first stage (50 kDa block length), followed by the final one-third containing the entire portion of EGDMA in the second stage. The resulting M50-Bz150-Bz225/E0.5 sample again formed homogeneous microparticles, but by contrast to the one-stage analogue, was also revealed to have retained a welldefined internal lamellar morphology (Fig. 4c). Furthermore, DSC analysis showed that the $T_{\mathrm{g}}$ of the PBzMA block increased by $\sim{ }^{\circ} \mathrm{C}$ when polymerised with EGDMA (66.3 ${ }^{\circ} \mathrm{C}$ versus $69.1{ }^{\circ} \mathrm{C}$ for M50-Bz50 and M50-Bz150-Bzz25/E0.5, respectively), while that of the PMMA block remained unchanged $\left(123.4{ }^{\circ} \mathrm{C}\right.$ versus $123.3{ }^{\circ} \mathrm{C}$ for M50-Bz50/E0.5 and M50-Bz50/E0.5, respectively) (SI Fig. 8). Although this is a greater increase than that observed for the P4VP domains within the PMMA- $b$-P4VP BCP crosslinked with $0.5 \mathrm{wt} \% \mathrm{DVB}$, the same general trend is followed, further corroborating that the PBzMA domains have been selectively crosslinked.
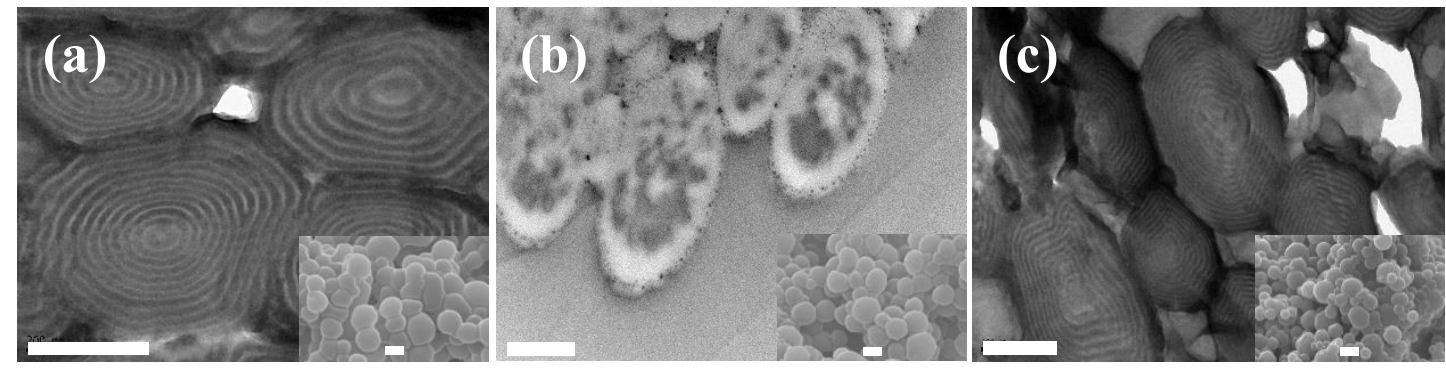

Figure 4. TEM cross-sectional views of the in situ crosslinked PMMA- $b$-PBzMA microparticles via RAFT dispersion polymerisation in $\mathrm{scCO}_{2}$. (a) non-crosslinked M50-Bz50; 


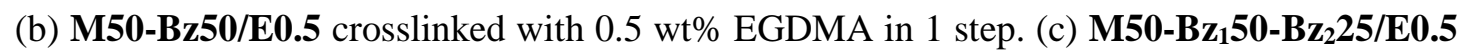
crosslinked with $0.5 \mathrm{wt} \%$ EGDMA in 2 steps. The sections were stained with $\mathrm{RuO}_{4}$ vapour for 4 hours, causing the PBzMA domains to appear dark in the images. The insets are the corresponding SEM images. The scale bars represent $500 \mathrm{~nm}$ and $1 \mu \mathrm{m}$ in the insets, respectively.

\subsection{Porosity control by degree of crosslinking}

We recently developed a facile approach to convert nanostructured BCP microparticles into porous microparticles via swelling/deswelling in alcohol. ${ }^{34}$ It was demonstrated that the porosity can be tailored over a wide size range and through diverse morphologies by adjusting the block ratio and block length. However, for this process to be successful, the swollen minority P4VP-block of PMMA- $b$-P4VP has to be limited to less than $35 \mathrm{~mol} \%$ to achieve porous microparticles without causing inter-particle fusion. This could be improved slightly through the use of a poorer swelling solvent, but ultimately restricts the types of BCP microparticles that are compatible with this process. Thus, we propose here that the porosity in these systems during the swelling/deswelling process could be tuned by the swelling degree of the minority block in response to the degree of crosslinking.

To examine the effect of crosslinking on the porosity generated during solvent swelling, PMMA- $b$-P4VP microparticles with varying levels of crosslinker $(0-8 \mathrm{wt} \%$ DVB) (Table 1) were exposed to a swelling/deswelling process in ethanol and hexane, respectively. Here, ethanol is good solvent for P4VP and hexane is non-solvent for both blocks. The non-crosslinked microparticles (M50-V33, Fig. 3a) developed large surface macropores of $\mathrm{d}_{\mathrm{w}}$ (pore width) $>100 \mathrm{~nm}$, and some spherical pores merged into interconnected pore channels. Moreover, the porous microparticles themselves were considerably fused, which results from swelling of the P4VP domains along with the collapse of the surrounding PMMA framework for microparticles with high P4VP ratios, as demonstrated in our recent report. ${ }^{34}$

After ethanol swelling, the minimally crosslinked (0.5 wt\% DVB) sample M50V33/D0.5 had a pore size of $d_{w}=\sim 20 \mathrm{~nm}$ (Fig. 5b), which is significantly smaller in comparison to the non-crosslinked M50-V33 sample $\left(d_{w}>100 \mathrm{~nm}\right)$ (Fig. 5a). M50V33/D0.5 also developed into perfectly discrete porous microparticles without interparticle fusion. When the crosslinking was increased a little further to $1 \mathrm{wt} \% \mathrm{DVB}$, 
(M50-V117-V 216/D1, Fig. 5c), the pore size decreased even further into the sub-10 nm range, and some were nearly closed. Critically, the M50-V17-V216/D4 sample synthesised with $4 \mathrm{wt} \%$ of crosslinker is almost entirely devoid of pores (Fig. 5d), and further increasing the DVB content to $8 \mathrm{wt} \%\left(\mathbf{M 5 0}-\mathbf{V}_{120} \mathbf{2} \mathbf{2} \mathbf{1 3} / \mathbf{D 8}\right)$ offers non-porous particles after ethanol swelling (SI Fig. 9). Overall these results highlights that the swelling of the P4VP domains in ethanol can be systematically reduced by increasing the quantity of DVB added during the polymerisation, particularly between the range of 0.5 to $4 \mathrm{wt} \%$.
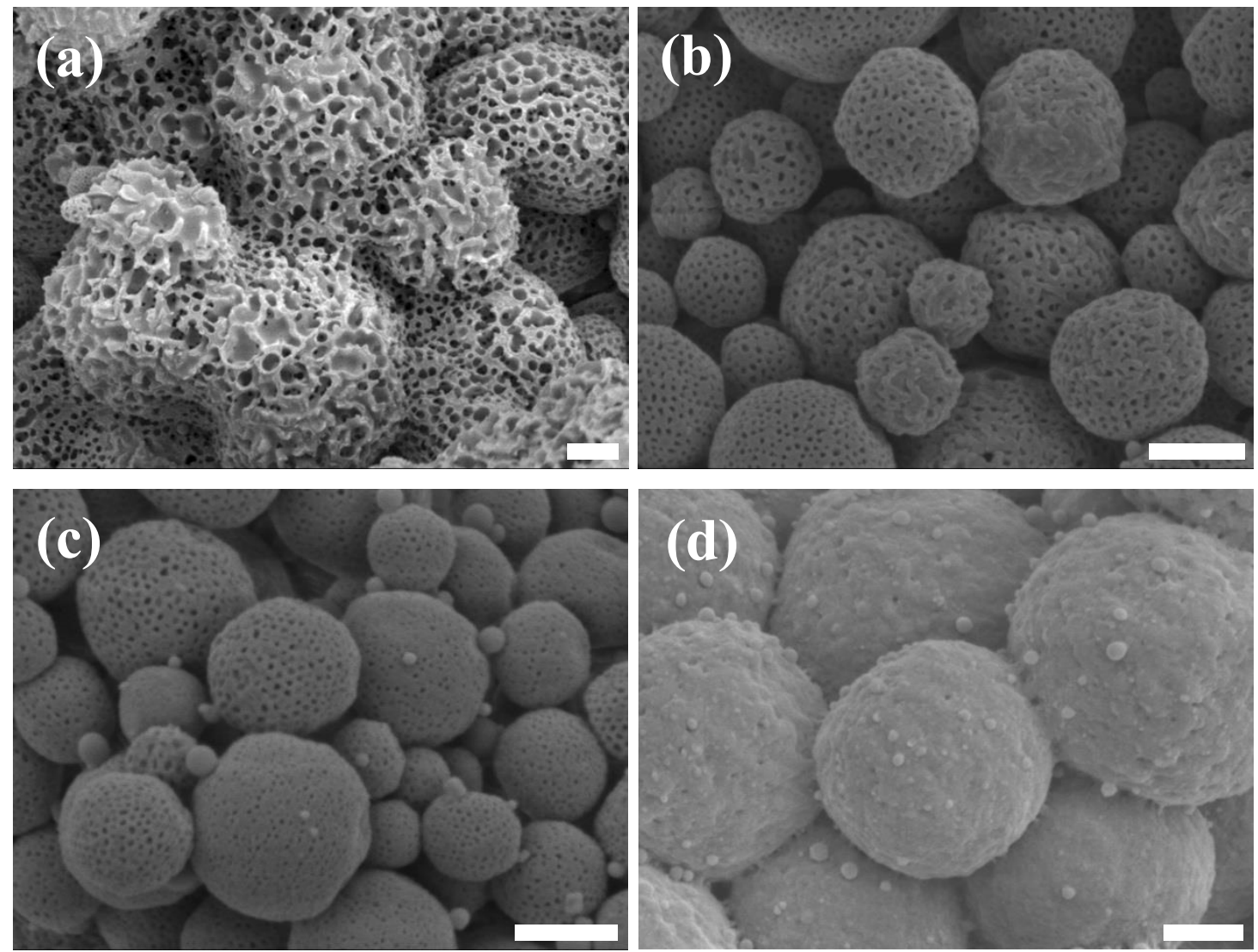

Figure 5. SEM images of crosslinked PMMA- $b$-P4VP microparticles after swelling in ethanol, which shows the porosity decreases remarkably as crosslinking increases. (a) non-crosslinked M50-V33; (b) M50-V33/D0.5 with 0.5 wt $\%$ DVB; (c) M50-V $\mathbf{1} 17-V_{2} 16 / D 1$ with 1 wt\% DVB; (d) M50-V $\mathbf{V}_{\mathbf{1}} \mathbf{1 7}-\mathbf{V}_{\mathbf{2}} \mathbf{1 6} / \mathbf{D 4}$ with $4 \mathrm{wt} \%$ DVB. The scale bar represents $500 \mathrm{~nm}$.

We have previously investigated the pore generation mechanism in PMMA- $b$-P4VP microparticles during the swelling/deswelling process, and clarified that the porosity is formed in the places previously occupied by the swollen minority block. In the case of the crosslinked microparticles, the swelling volume of the P4VP domains in ethanol decreases as a function of the DVB content, thus shrinking the voids generated from 
the collapsing P4VP chains. Byard et. al studied the swelling of crosslinked poly $(\mathrm{N}, \mathrm{N}-$ dimethyl acrylamide)-block-poly(diacetone acrylamide) (PDMAm-b-PDAAm) vesicles crosslinked with adipic acid dihydrazide (ADH) ${ }^{48}$ They found that substantial swelling in methanol was observed for the lightly crosslinked vesicles. In contrast, much less swelling occurred for $\mathrm{ADH} / \mathrm{DAAM} \geq 0.050$, because more extensive crosslinking was obtained under these conditions. Moreover, a maximum covalent stabilisation was achieved for $\mathrm{ADH} / \mathrm{DAAM} \geq 0.075$. Our results are consistent with these observations. The swelling substantially decreased as the DVB crosslinker level increased from 0.5 to $4 \mathrm{wt} \%$, at which point the maximum resistance to swelling was achieved (non-porosity), providing an alternative methodology for tuning porosity control during swelling in addition to varying the BCP molecular weights and/or block ratios.

To further examine the internal porosity of the crosslinked microparticles after swelling/deswelling, TEM analysis was carried out. Fig. 6a shows that the noncrosslinked M50-V33 sample has macropores > $50 \mathrm{~nm}$ and extensive inter-particle fusion, which is consistent with the SEM observations. At 0.5 wt\% DVB, M50V33/D0.5 shows an intricate porous structure that has inter-connected channels throughout the entirety of each microparticle (Fig. 6b). Furthermore, the pore size decreased remarkably, with $\mathrm{d}_{\mathrm{w}} \sim 20 \mathrm{~nm}$, and inter-particle fusion is completely avoided.
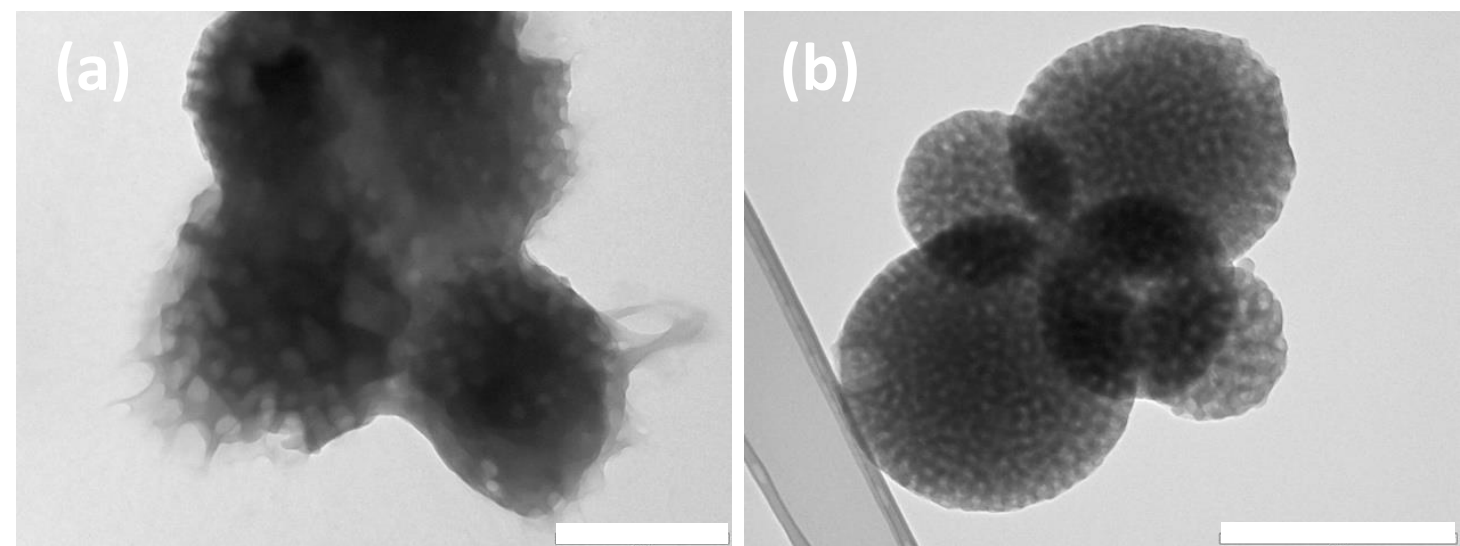

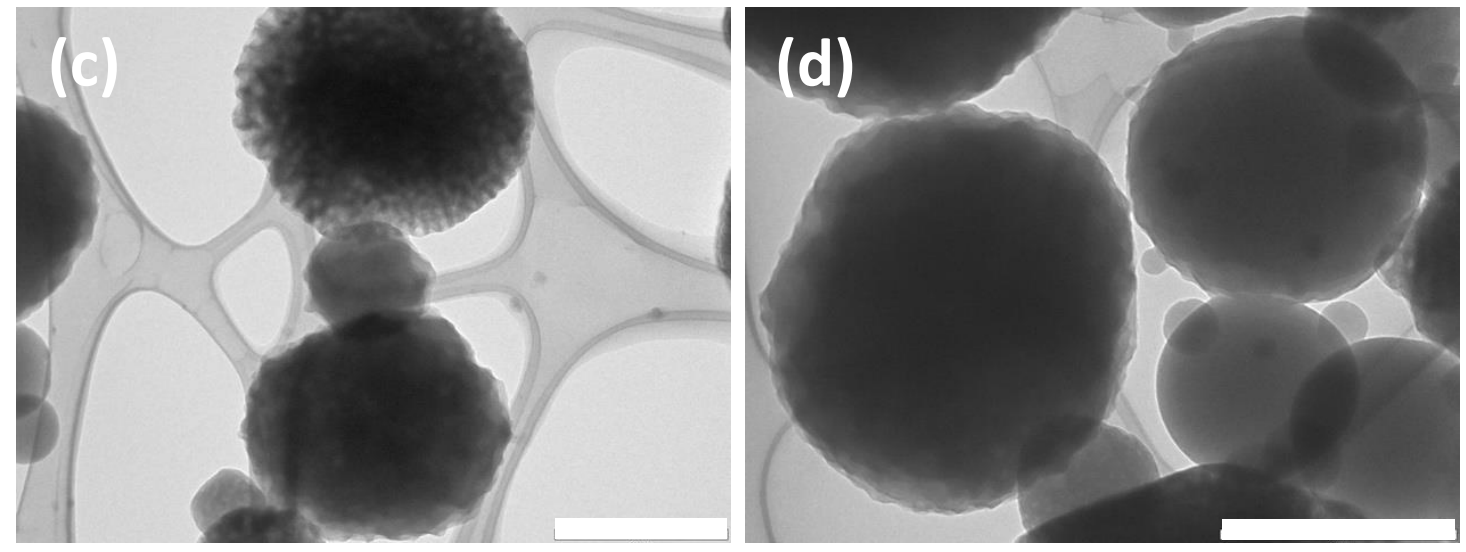

Figure 6. TEM images of the crosslinked PMMA- $b$-P4VP microparticles after swelling in ethanol, which shows the porosity decreases as crosslinker increases. (a) non-crosslinked sample M50-V33; (b) M50-V33/D0.5 with 0.5 wt\% DVB; (c) M50-V 1 17-V $\mathbf{2} 16 / D 1$ with 1 wt\% DVB; (d) M50-V $\mathbf{V}_{\mathbf{1}} \mathbf{1 7}-\mathbf{V}_{\mathbf{2}} \mathbf{1 6} / \mathbf{D 4}$ with 4 wt\% DVB. The samples had no staining. The scale bar represents $500 \mathrm{~nm}$.

For the samples synthesised with 1 wt\% DVB $\left(\mathbf{M 5 0}-V_{1} \mathbf{1 7}-\mathbf{V}_{\mathbf{2}} \mathbf{1 6} / \mathbf{D 1}\right)$, the porosity decreased further and some microparticles appear to lack porosity (Fig. 6c). These nonporous particles are observed exclusively when the DVB content is increased to $4 \mathrm{wt} \%$ (Fig. 6d for M50-V117-V216/D4). These results further corroborate the SEM observations that the pore size is remarkably reduced at a higher DVB content - from macropores, to mesopores of $\sim 20 \mathrm{~nm}$, to sub-10 nm, and finally to non-porous.

Interestingly, the swelling behaviour (or porosity) of crosslinked M50-V33/D0.5 in ethanol is analogous to the non-crosslinked sample M50-V12.4, whilst M50-V117V216/D1 is analogous to M50-V7.6 (M50-V12.4 and M50-V7.6 are referred to as MV19.9 and M-V13.3, respectively, in our recent report). ${ }^{34}$ Compared to manipulating the block length, crosslinking has a similar effect for porosity control during the swelling/deswelling process. Smaller sized pores can therefore be obtained by either increasing crosslinking or decreasing the block length. Overall, the possibility of combining these approaches will greatly improve the versatility of this microparticle platform for generating hierarchically porous materials.

\subsection{Nano-mapping the swelling of crosslinked P4VP domains in humidity}

To visualise the swelling behaviour the of P4VP nano-domains within a 3-dimensional microparticle in situ, AFM mapping of thin sections of the BCP microparticles ( $~ 80$ 
$\mathrm{nm}$ ) in controlled humidity was carried out. Fig. 7 shows the monitoring of the swelling of non-crosslinked M50-V33 microparticle sections in humidity. The darker spherical areas in the height image are recognised as the P4VP domains. After swelling in $80 \%$ humidity for $1 \mathrm{~h}$, the spherical P4VP domains develop into brighter areas than the PMMA matrix (Fig. 7b). The height profiles for a local area as line-marked in Fig. 7a,b distinctly illustrate that the P4VP domains dramatically increase in height, to $12 \mathrm{~nm}$ and $20 \mathrm{~nm}$ for the P4VP domains with $\mathrm{d}$ (domain size) $=100 \mathrm{~nm}$ and $125 \mathrm{~nm}$, respectively (Fig. 7c,d). P4VP is a hydrophobic polymer that is insoluble in water until more than ca. $35 \%$ of the pyridine groups are charged, e.g., by protonation. ${ }^{49}$ These AFM results confirm that non-crosslinked and unprotonated P4VP domains are readily swelled by water.

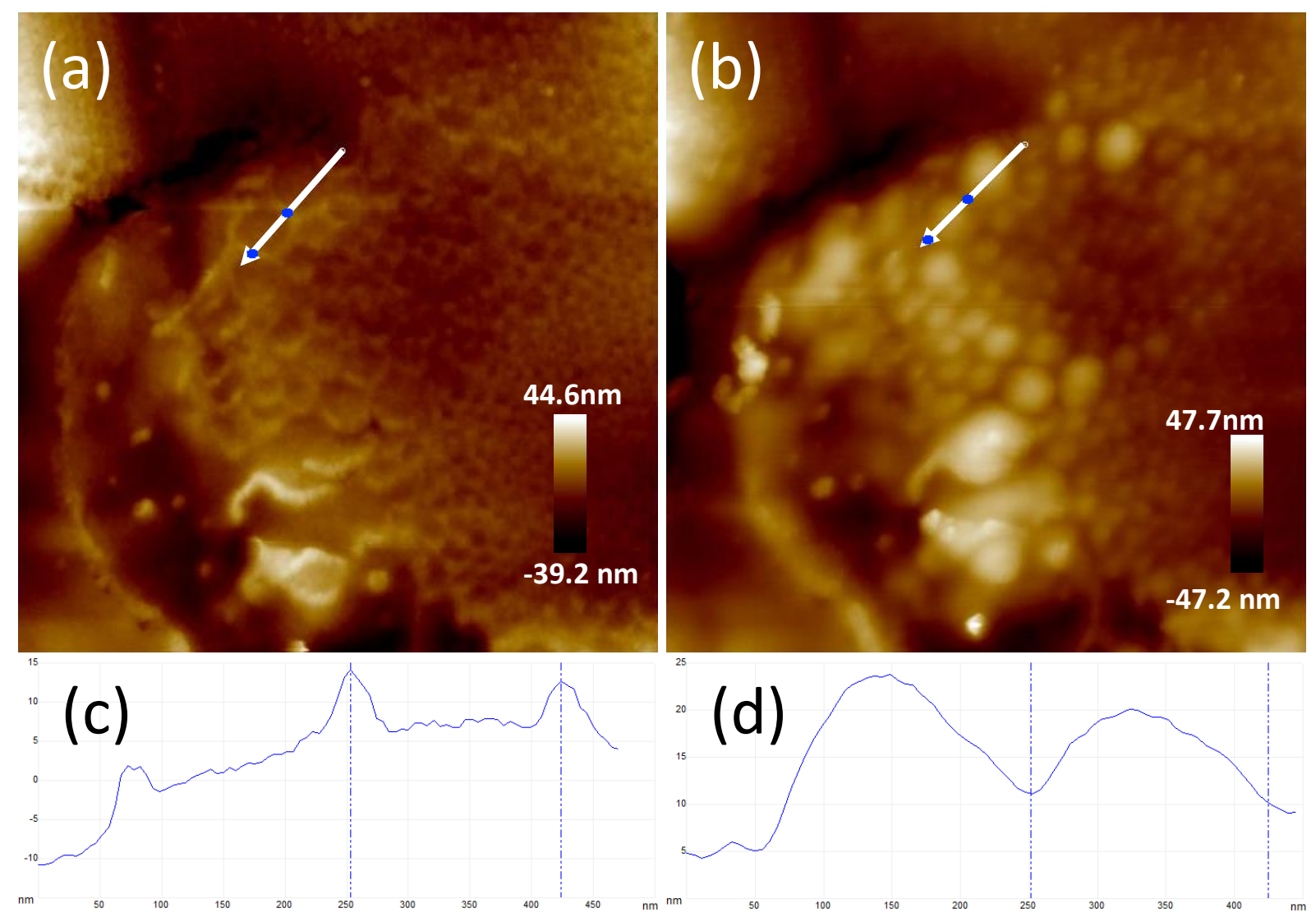

Figure 7. AFM in situ monitoring of the swelling behaviour of nano-domains of noncrosslinked M50-V33 microparticles (thin sections $\sim 80 \mathrm{~nm}$ on TEM copper grids). (a) original sample, (b) after re-swelling in humidity of $80 \%$ for 1 hour, (c,d) height profiles of sections as line marked in $(a, b)$, respectively. The image is $2 \times 2 \mu \mathrm{m}^{2}$.

By comparison, the nano-mapping data of the crosslinked microparticle sections show that the P4VP domains of M50-V120-V $\mathbf{V}_{2} 13 / D 8$ display no obvious change in height 
relative to the PMMA matrix after treatment in $80 \%$ humidity (Fig. 8). This further confirms that crosslinking the P4VP domains of PMMA- $b$-P4VP BCPs renders them highly resistant to swelling in the presence of water, and presumably other solvents by extension.
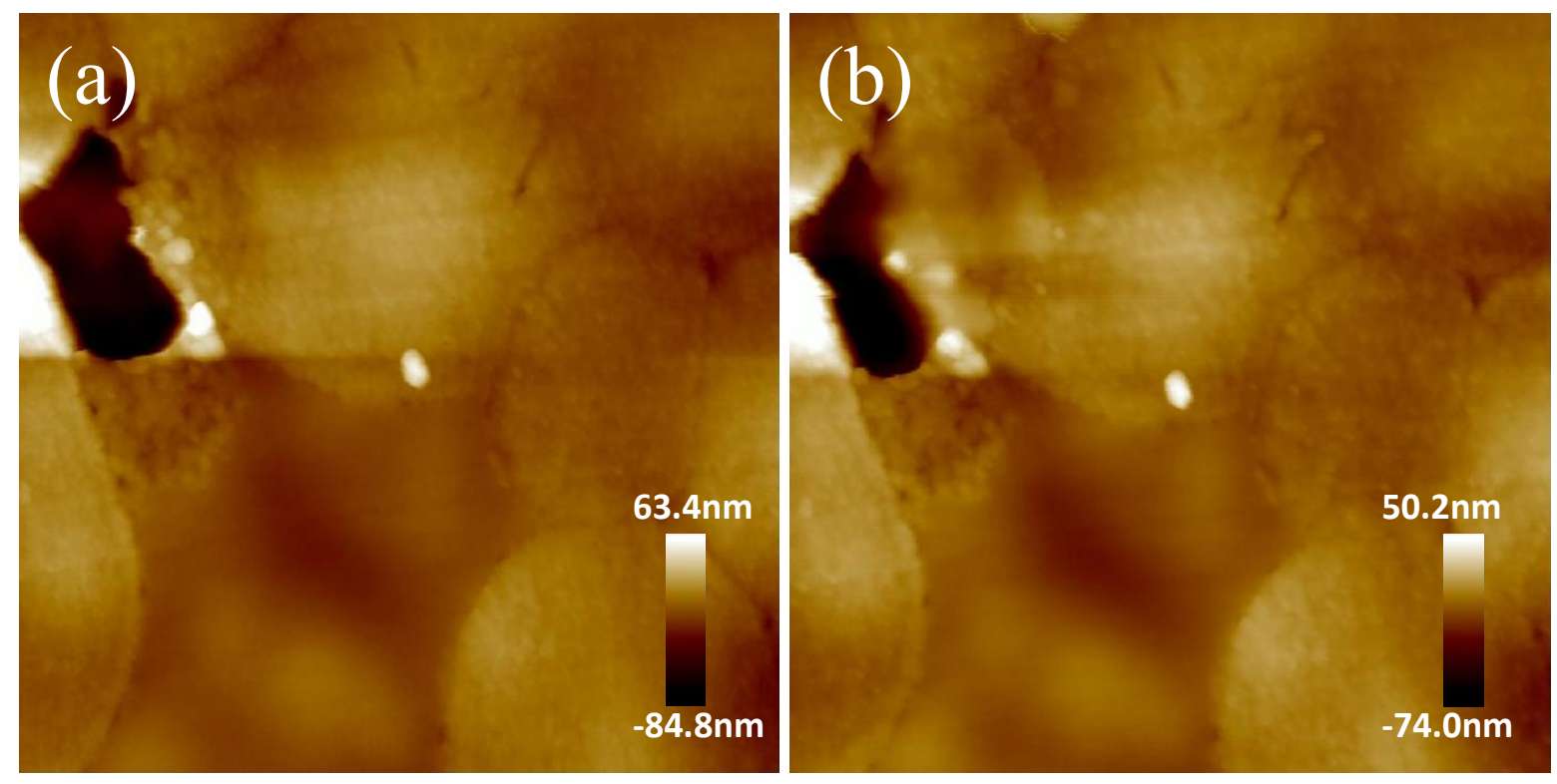

Figure 8. AFM in situ monitoring of the swelling of crosslinked $\mathbf{M 5 0}-\mathrm{V}_{\mathbf{1}} \mathbf{2 0}-\mathrm{V}_{\mathbf{2}} \mathbf{1 3} / \mathrm{D8}$ microparticles ( $8 \mathrm{wt} \%$ DVB) (thin sections $\sim 80 \mathrm{~nm}$ on TEM copper grids) in humidity. (a) original sample, (b) after re-swelling in $80 \%$ humidity for 1 hour. The image is $2 \times 2 \mu \mathrm{m}^{2}$.

\section{Conclusions}

We present a new approach for introducing crosslinking into nanostructured $\mathrm{BCP}$ microparticles via in situ crosslinking copolymerisation, as a means of achieving and retaining structural integrity under solvated conditions. Delaying the addition of the crosslinker DVB and a portion of the 4VP monomer enables the growing chains of the precursor PMMA- $b$-P4VP microparticles to be crosslinked, while also preserving the polymerisation induced microphase separated morphology within the microparticles. Critically, a high crosslinker level of $8 \mathrm{wt} \%$ DVB can be added to the reaction in this way to produce microparticles with highly crosslinked nanopatterns. Moreover, this synthetic protocol is shown to be applicable to a second BCP system, enabling crosslinked PMMA- $b$-PBzMA microparticles with an internal lamellar morphology to be achieved.

The structural stability of the crosslinked PMMA- $b$-P4VP microparticles was first 
demonstrated through dispersion into good solvents, after which both the internal nanostructures and microparticulate scaffolds were preserved. Notably, the porosity generated by swelling in ethanol is found to be well controlled by the quantity of crosslinker added to the polymerisation. Macropores $>100 \mathrm{~nm}$, to mesopores of $\sim 20$ $\mathrm{nm}$, to sub-10 $\mathrm{nm}$ pores, and finally non-porous structures were all achieved by increasing the level of DVB from $0,0.5,1$, to $4 \mathrm{wt} \%$, respectively. The $T_{\mathrm{g}}$ values of the crosslinked P4VP domains were also found to increase as a function of the quantity of DVB added, which also coincided with a progressive decrease in the microparticle diameters. In situ AFM nano-mapping studies of the non-crosslinked and crosslinked P4VP domains in $80 \%$ humidity revealed that highly crosslinked PMMA- $b$-P4VP microparticles ( $8 \mathrm{wt} \%$ DVB) are completely immune to swelling by moisture, in contrast to their non-crosslinked analogues.

This versatile approach further expands the available repertoire for fabricating porous BCP microparticles with tunable physico-chemical properties, morphologies and pore sizes, greatly broadening their application potential in various fields.

\section{Supporting Information}

${ }^{1} \mathrm{H}$ NMR and GPC data of the non-crosslinked BPC samples; SEM and TEM images of M50-V33/D1, M50- $\mathrm{V}_{1} 17-\mathrm{V}_{2} 16 / \mathrm{D} 2$ and $M 50-\mathrm{V}_{1} 17-\mathrm{V}_{2} 16 / \mathrm{D} 4$; photographs of the various BCPs dispersed in chloroform; TEM images of M50-V33 and M50-V33/D0.5 dissolved in THF at a concentration of $2 \mathrm{mg} / \mathrm{ml}$; DSC traces for the crosslinked PMMA- $b$-P4VP and PMMA- $b$-PBzMA samples prepared via the two-step method; SEM image of M50-V120$\mathbf{V}_{\mathbf{2}}$ 13/D8 microparticles after swelling in ethanol . This material is available free of charge via the Internet at http://...

\section{Notes}

The authors declare no competing financial interest.

\section{Acknowledgements}

We are grateful to the Leverhulme Trust (RPG-2014-034) for their support of this project ( $\mathrm{GH}$ and $\mathrm{TMB})$. KA thanks the Malaysian Rubber Board for her $\mathrm{PhD}$ Scholarship. STS is grateful to the Erasmus+ scheme for the award of a higher 
education traineeship to support his visit to Nottingham. MA acknowledges the UNNES-IDB for his PhD Scholarship to Nottingham. We acknowledge the invaluable technical support received from our high pressure workshop technicians Richard Wilson, Peter Fields and Martin Dellar. Our research would not have been possible without the excellent electron microscopy facilities at the Nanoscale and Microscale Research Centre (NMRC) within the University of Nottingham, in particular the JEOL 7100F FEG-SEM and FEI Tecnai BioTwin-12 TEM. We thank also Ms Nicola Weston for her assistance with electron microscopy. We thank Prof. Ullrich Steiner for his input on BCP materials.

\section{References}

(1) Robb, M. J.; Connal, L. A.; Lee, B. F.; Lynd, N. A.; Hawker, C. J. Polym. Chem. 2012, 3, 16181628.

(2) Park, M.-K.; Jun, S.; Kim, I.; Jin, S.-M.; Kim, J.-G.; Shin, T. J.; Lee, E. Adv. Funct. Mater. 2015, 25, 4570-4579.

(3) Nykänen, A.; Rahikkala, A.; Hirvonen, S.-P.; Aseyev, V.; Tenhu, H.; Mezzenga, R.; Raula, J.; Kauppinen, E.; Ruokolainen, J. Macromolecules 2012, 45, 8401-8411.

(4) Rahikkala, A.; Aseyev, V.; Tenhu, H.; Kauppinen, E. I.; Raula, J. Biomacromolecules 2015, 16, 2750-2756.

(5) Klinger, D.; Wang, C. X.; Connal, L. A.; Audus, D. J.; Jang, S. G.; Kraemer, S.; Killops, K. L.; Fredrickson, G. H.; Kramer, E. J.; Hawker, C. J. Angew. Chem., Int. Ed. 2014, 53, 7018-7022.

(6) Zhu, Y.; Gao, X.; Luo, Y. J. Appl. Polym. Sci. 2016, 133.

(7) Yu, H.; Qiu, X.; Nunes, S. P.; Peinemann, K.-V. Nat. Commun. 2014, 5.

(8) Bennett, T. M.; He, G.; Larder, R. R.; Fischer, M. G.; Rance, G. A.; Fay, M. W.; Pearce, A. K.; Parmenter, C. D. J.; Steiner, U.; Howdle, S. M. Nano Lett. 2018, 18, 7560-7569.

(9) Fischer, M. G.; Hua, X.; Wilts, B. D.; Gunkel, I.; Bennett, T. M.; Steiner, U. ACS Appl. Mater. Interfaces 2017, 9, 22388-22397.

(10) Prodan, E.; Radloff, C.; Halas, N. J.; Nordlander, P. Science 2003, 302, 419-422.

(11) Stefik, M.; Guldin, S.; Vignolini, S.; Wiesner, U.; Steiner, U. Chem. Soc. Rev. 2015, 44, 50765091.

(12) Kitayama, Y.; Kagawa, Y.; Minami, H.; Okubo, M. Langmuir 2010, 26, 7029-7034.

(13) Monteiro, M. J.; de Barbeyrac, J. Macromolecules 2001, 34, 4416-4423.

(14) Jennings, J.; Beija, M.; Richez, A. P.; Cooper, S. D.; Mignot, P. E.; Thurecht, K. J.; Jack, K. S.; Howdle, S. M. J. Am. Chem. Soc. 2012, 134, 4772-4781.

(15) Jennings, J.; Beija, M.; Kennon, J. T.; Willcock, H.; O'Reilly, R. K.; Rimmer, S.; Howdle, S. M. Macromolecules 2013, 46, 6843-6851.

(16) Froimowicz, P.; van Heukelum, B.; Scholten, C.; Greiner, K.; Araujo, O.; Landfester, K. J. Polym. Sci., Part A: Polym. Chem. 2014, 52, 883-889.

(17) Guimarães, T. R.; Khan, M.; Kuchel, R. P.; Morrow, I. C.; Minami, H.; Moad, G.; Perrier, S.; Zetterlund, P. B. Macromolecules 2019, 52, 2965-2974.

(18) Lu, J.; Owen, S. C.; Shoichet, M. S. Macromolecules 2011, 44, 6002-6008.

(19) Wang, X.; Liu, G.; Hu, J.; Zhang, G.; Liu, S. Angew. Chem., Int. Ed. 2014, 53, 3138-3142.

(20) Chambon, P.; Blanazs, A.; Battaglia, G.; Armes, S. P. Langmuir 2012, 28, 1196-1205.

(21) Thompson, K. L.; Chambon, P.; Verber, R.; Armes, S. P. J. Am. Chem. Soc. 2012, 134, 1245012453. 
(22) Discher, B. M.; Bermudez, H.; Hammer, D. A.; Discher, D. E.; Won, Y. Y.; Bates, F. S. J. Phys. Chem. B 2002, 106, 2848-2854.

(23) Henderson, I. M.; Quintana, H. A.; Martinez, J. A.; Paxton, W. F. Chem. Mater. 2015, 27, 4808-4813.

(24) Gaitzsch, J.; Appelhans, D.; Wang, L.; Battaglia, G.; Voit, B. Angew. Chem., Int. Ed. 2012, 51, 4448-4451.

(25) Xu, X.; Flores, J. D.; McCormick, C. L. Macromolecules 2011, 44, 1327-1334.

(26) Qiu, L.; Xu, C.-R.; Zhong, F.; Hong, C.-Y.; Pan, C.-Y. Macromol. Chem. Phys. 2016, 217, 10471056.

(27) Zhou, W.; Qu, Q.; Yu, W.; An, Z. ACS Macro Lett. 2014, 3, 1220-1224.

(28) Lovett, J. R.; Ratcliffe, L. P. D.; Warren, N. J.; Armes, S. P. Macromolecules 2016, 49, 29282941.

(29) Qiu, J.; Charleux, B.; Matyjaszewski, K. Prog. Polym. Sci. 2001, 26, 2083-2134.

(30) Sugihara, S.; Armes, S. P.; Blanazs, A.; Lewis, A. L. Soft Matter 2011, 7, 10787-10793.

(31) Chambon, P.; Blanazs, A.; Battaglia, G.; Armes, S. P. Macromolecules 2012, 45, 5081-5090.

(32) Qu, Q.; Liu, G.; Lv, X.; Zhang, B.; An, Z. ACS Macro Lett. 2016, 5, 316-320.

(33) Zhang, L.; Lu, Q.; Lv, X.; Shen, L.; Zhang, B.; An, Z. Macromolecules 2017, 50, 2165-2174.

(34) He, G.; Bennett, T. M.; Alauhdin, M.; Fay, M. W.; Liu, X.; Schwab, S. T.; Sun, C.-g.; Howdle, S. M. Polym. Chem. 2018, 9, 3808-3819.

(35) Alauhdin, M.; Bennett, T. M.; He, G.; Bassett, S. P.; Portale, G.; Bras, W.; Hermida-Merino, D.; Howdle, S. M. Polym. Chem. 2019.

(36) McAllister, T. D.; Farrand, L. D.; Howdle, S. M. Macromol. Chem. Phys. 2016, 217, 22942301.

(37) Lai, J. T.; Filla, D.; Shea, R. Macromolecules 2002, 35, 6754-6756.

(38) Jennings, J.; Beija, M.; Kennon, J. T.; Willcock, H.; O’Reilly, R. K.; Rimmer, S.; Howdle, S. M. Macromolecules 2013, 46, 6843-6851.

(39) Gregory, A. M.; Thurecht, K. J.; Howdle, S. M. Macromolecules 2008, 41, 1215-1222.

(40) Truong, N. P.; Dussert, M. V.; Whittaker, M. R.; Quinn, J. F.; Davis, T. P. Polym. Chem. 2015, 6, 3865-3874.

(41) Song, J.-S.; Winnik, M. A. Macromolecules 2005, 38, 8300-8307.

(42) Varshney, S. K.; Zhong, X. F.; Eisenberg, A. Macromolecules 1993, 26, 701-706.

(43) Lee, J.; Hogen-Esch, T. E. Macromolecules 2001, 34, 2805-2811.

(44) Shin, J.; Bae, W.; Kim, H. Colloid Polym. Sci. 2010, 288, 271-282.

(45) Acar, N. J. Appl. Polym. Sci. 2001, 81, 2609-2614.

(46) Jennings, J.; Bassett, S. P.; Hermida-Merino, D.; Portale, G.; Bras, W.; Knight, L.; Titman, J. J.; Higuchi, T.; Jinnai, H.; Howdle, S. M. Polym. Chem. 2016, 7, 905-916.

(47) Matsen, M. W.; Bates, F. S. Macromolecules 1996, 29, 1091-1098.

(48) Byard, S. J.; Williams, M.; McKenzie, B. E.; Blanazs, A.; Armes, S. P. Macromolecules 2017, 50, 1482-1493.

(49) Satoh, M.; Yoda, E.; Hayashi, T.; Komiyama, J. Macromolecules 1989, 22, 1808-1812. 